\title{
On sub-determinants and the diameter of polyhedra*
}

\author{
Nicolas Bonifas ${ }^{\dagger} \quad$ Marco Di Summa $^{\ddagger} \quad$ Friedrich Eisenbrand $^{\S} \quad$ Nicolai Hähnle $^{\llbracket}$ \\ Martin Niemeier"
}

August 28, 2018

\begin{abstract}
We derive a new upper bound on the diameter of a polyhedron $P=\left\{x \in \mathbb{R}^{n}: A x \leqslant b\right\}$, where $A \in$ $\mathbb{Z}^{m \times n}$. The bound is polynomial in $n$ and the largest absolute value of a sub-determinant of $A$, denoted by $\Delta$. More precisely, we show that the diameter of $P$ is bounded by $O\left(\Delta^{2} n^{4} \log n \Delta\right)$. If $P$ is bounded, then we show that the diameter of $P$ is at most $O\left(\Delta^{2} n^{3.5} \log n \Delta\right)$.

For the special case in which $A$ is a totally unimodular matrix, the bounds are $O\left(n^{4} \log n\right)$ and $O\left(n^{3.5} \log n\right)$ respectively. This improves over the previous best bound of $O\left(m^{16} n^{3}(\log m n)^{3}\right)$ due to Dyer and Frieze [DF94].
\end{abstract}

\section{Introduction}

One of the fundamental open problems in optimization and discrete geometry is the question whether the diameter of a polyhedron can be bounded by a polynomial in the dimension and the number of its defining inequalities. The problem is readily explained: A polyhedron is a set of the form $P=\left\{x \in \mathbb{R}^{n}: A x \leqslant b\right\}$, where $A \in \mathbb{R}^{m \times n}$ is a matrix and $b \in \mathbb{R}^{m}$ is an $m$-dimensional vector. A vertex of $P$ is a point $x^{*} \in P$ such that there exist $n$ linearly independent rows of $A$ whose corresponding inequalities of $A x \leqslant b$ are satisfied by $x^{*}$ with equality. Throughout this paper, we assume that the polyhedron $P$ is pointed, i.e. it has vertices, which is equivalent to saying that the matrix $A$ has full column-rank. Two different vertices $x^{*}$ and $y^{*}$ are neighbors if they are the endpoints of an edge of the polyhedron, i.e. there exist $n-1$ linearly independent rows of $A$ whose corresponding inequalities of $A x \leqslant b$ are satisfied with equality both by $x^{*}$ and $y^{*}$. In this way, we obtain the undirected polyhedral graph with edges being pairs of neighboring vertices of $P$. This graph is connected. The diameter of $P$ is the smallest natural number that bounds the length of a shortest path between any pair of vertices in this graph. The question is now as follows:

Can the diameter of a polyhedron $P=\left\{x \in \mathbb{R}^{n}: A x \leqslant b\right\}$ be bounded by a polynomial in $m$ and $n$ ?

The belief in a positive answer to this question is called the polynomial Hirsch conjecture. Despite a lot of research effort during the last 50 years, the gap between lower and upper bounds on the diameter remains huge. While, when the dimension $n$ is fixed, the diameter can be bounded by a

\footnotetext{
${ }^{*}$ An extended abstract of this paper was presented at the 28-th annual ACM symposium on Computational Geometry (SOCG 12)

${ }^{\dagger}$ LIX, École Polytechnique, Palaiseau and IBM, Gentilly (France). bonifas@lix.polytechnique.fr

${ }^{\ddagger}$ Dipartimento di Matematica, Università di Padova (Italy). disumma@math. unipd. it

${ }^{\S}$ Ecole Polytechnique Fédérale de Lausanne (Switzerland). friedrich.eisenbrand@epf 1 .ch

"University of Bonn (Germany). haehnle@or . uni-bonn . de

${ }^{\|}$Technische Universität Berlin (Germany). martin.niemeier@tu-berlin.de
} 
linear function of $m$ [Lar70, Bar74], for the general case the best upper bound, due to Kalai and Kleitman [KK92], is $m^{1+\log n}$. The best lower bound is of the form $(1+\varepsilon) \cdot m$ for some $\varepsilon>0$ in fixed and sufficiently large dimension $n$. This is due to a celebrated result of Santos [San12] who disproved the, until then longstanding, original Hirsch conjecture for polytopes. The Hirsch conjecture stated that the diameter of a bounded polyhedron 11 is at most $m-n$. Interestingly, this huge gap (polynomial versus quasi-polynomial) is also not closed in a very simple combinatorial abstraction of polyhedral graphs [EHRR10]. However, it was shown by Vershynin [Ver09] that every polyhedron can be perturbed by a small random amount so that the expected diameter of the resulting polyhedron is bounded by a polynomial in $n$ and $\ln m$. See Kim and Santos [KS10] for a recent survey.

In light of the importance and apparent difficulty of the open question above, many researchers have shown that it can be answered in an affirmative way in some special cases. Naddef [Nad89] proved that the Hirsch conjecture holds true for 0/1-polytopes. Orlin [Orl97 provided a quadratic upper bound for flow-polytopes. Brightwell et al. BvdHS06] showed that the diameter of the transportation polytope is linear in $m$ and $n$, and a similar result holds for the dual of a transportation polytope [Bal84] and the axial 3-way transportation polytope [DLKOS09].

The results on flow polytopes and classical transportation polytopes concern polyhedra defined by totally unimodular matrices, i.e., integer matrices whose sub-determinants are $0, \pm 1$. For such polyhedra Dyer and Frieze [DF94] had previously shown that the diameter is bounded by a polynomial in $n$ and $m$. Their bound is $O\left(m^{16} n^{3}(\log m n)^{3}\right)$. Their result is also algorithmic: they show that there exists a randomized simplex-algorithm that solves linear programs defined by totally unimodular matrices in polynomial time.

Our main result is a generalization and considerable improvement of the diameter bound of Dyer and Frieze. We show that the diameter of a polyhedron $P=\left\{x \in \mathbb{R}^{n}: A x \leqslant b\right\}$, with $A \in \mathbb{Z}^{m \times n}$ is bounded by $O\left(\Delta^{2} n^{4} \log n \Delta\right)$. Here, $\Delta$ denotes the largest absolute value of a sub-determinant of $A$. If $P$ is bounded, i.e., a polytope, then we can show that the diameter of $P$ is at most $O\left(\Delta^{2} n^{3.5} \log n \Delta\right)$. To compare our bound with the one of Dyer and Frieze one has to set $\Delta$ above to one and obtains $O\left(n^{4} \log n\right)$ and $O\left(n^{3.5} \log n\right)$ respectively. Notice that our bound is independent of $m$, i.e., the number of rows of $A$.

\section{The proof method}

Let $u$ and $v$ be two vertices of $P$. We estimate the maximum number of iterations of two breadthfirst-search explorations of the polyhedral graph, one initiated at $u$, the other initiated at $v$, until a common vertex is discovered. The diameter of $P$ is at most twice this number of iterations. The main idea in the analysis is to reason about the normal cones of vertices of $P$ and to exploit a certain volume expansion property.

We can assume that $P=\left\{x \in \mathbb{R}^{n}: A x \leqslant b\right\}$ is non-degenerate, i.e., each vertex has exactly $n$ tight inequalities. This can be achieved by slightly perturbing the right-hand side vector $b$ : in this way the diameter can only grow. Notice that the polyhedron is then also full-dimensional. We denote the polyhedral graph of $P$ by $G_{P}=(V, E)$. Let $v \in V$ now be a vertex of $P$. The normal cone $C_{\nu}$ of $v$ is the set of all vectors $c \in \mathbb{R}^{n}$ such that $v$ is an optimal solution of the linear program $\max \left\{c^{T} x: x \in \mathbb{R}^{n}, A x \leqslant b\right\}$. The normal cone $C_{v}$ of a vertex of $v$ is a full-dimensional simplicial polyhedral cone. Two vertices $v$ and $v^{\prime}$ are adjacent if and only if $C_{v}$ and $C_{v^{\prime}}$ share a facet. No two distinct normal cones share an interior point. Furthermore, if $P$ is a polytope, then the union of the normal cones of vertices of $P$ is the complete space $\mathbb{R}^{n}$.

We now define the volume of a set $U \subseteq V$ of vertices as the volume of the union of the normal

\footnotetext{
${ }^{1}$ A counterexample to the same conjecture for unbounded polyhedra was found in 1967 by Klee and Walkup [KW67.
} 
cones of $U$ intersected with the unit ball $B_{n}=\left\{x \in \mathbb{R}^{n}:\|x\|_{2} \leqslant 1\right\}$, i.e.,

$$
\operatorname{vol}(U):=\operatorname{vol}\left(\bigcup_{\nu \in U} C_{\nu} \cap B_{n}\right)
$$

Consider an iteration of breadth-first-search. Let $I \subseteq V$ be the set of vertices that have been discovered so far. Breadth-first-search will next discover the neighborhood of $I$, which we denote by $\mathscr{N}(I)$.

Together with the integrality of $A$, the bound $\Delta$ on the subdeterminants guarantees that the angle between one facet of a normal cone $C_{v}$ and the opposite ray is not too small. We combine this fact, which we formalize in Lemma 3 , with an isoperimetric inequality to show that the volume of $\mathscr{N}(I)$ is large relative to the volume of $I$.

Lemma 1. Let $P=\left\{x \in \mathbb{R}^{n}: A x \leqslant b\right\}$ be a polytope where all sub-determinants of $A \in \mathbb{Z}^{m \times n}$ are bounded by $\Delta$ in absolute value and let $I \subseteq V$ be a set of vertices of $G_{P}$ with $\operatorname{vol}(I) \leqslant(1 / 2) \cdot \operatorname{vol}\left(B_{n}\right)$. Then the volume of the neighborhood of $I$ is at least

$$
\operatorname{vol}(\mathscr{N}(I)) \geqslant \sqrt{\frac{2}{\pi}} \frac{1}{\Delta^{2} n^{2.5}} \cdot \operatorname{vol}(I) .
$$

We provide the proof of this lemma in the next section. Our diameter bound for polytopes is an easy consequence:

Theorem 2. Let $P=\left\{x \in \mathbb{R}^{n}: A x \leqslant b\right\}$ be a polytope where all subdeterminants of $A \in \mathbb{Z}^{m \times n}$ are bounded by $\Delta$ in absolute value. The diameter of $P$ is bounded by $O\left(\Delta^{2} n^{3.5} \log n \Delta\right)$.

Proof. We estimate the maximum number of iterations of breadth-first-search until the total volume of the discovered vertices exceeds $(1 / 2) \cdot \operatorname{vol}\left(B_{n}\right)$. This is an upper bound on the aforementioned maximum number of iterations of two breadth-first-search explorations until a common vertex is discovered.

Suppose we start at vertex $v$ and let $I_{j}$ be the vertices that have been discovered during the first $j$ iterations. We have $I_{o}=\{v\}$. If $j \geqslant 1$ and $\operatorname{vol}\left(I_{j-1}\right) \leqslant(1 / 2) \cdot \operatorname{vol}\left(B_{n}\right)$ we have by Lemma 1

$$
\begin{aligned}
\operatorname{vol}\left(I_{j}\right) & \geqslant\left(1+\sqrt{\frac{2}{\pi}} \frac{1}{\Delta^{2} n^{2.5}}\right) \operatorname{vol}\left(I_{j-1}\right) \\
& \geqslant\left(1+\sqrt{\frac{2}{\pi}} \frac{1}{\Delta^{2} n^{2.5}}\right)^{j} \operatorname{vol}\left(I_{0}\right) .
\end{aligned}
$$

The condition $\operatorname{vol}\left(I_{j}\right) \leqslant(1 / 2) \cdot \operatorname{vol}\left(B_{n}\right)$ implies

$$
\left(1+\frac{1}{\sqrt{\frac{\pi}{2}} \Delta^{2} n^{2.5}}\right)^{j} \operatorname{vol}\left(I_{0}\right) \leqslant 2^{n} .
$$

This is equivalent to

$$
j \cdot \ln \left(1+\frac{1}{\sqrt{\frac{\pi}{2}} \Delta^{2} n^{2.5}}\right) \leqslant \ln \left(2^{n} / \operatorname{vol}\left(I_{0}\right)\right) .
$$

For $0 \leqslant x \leqslant 1$ one has $\ln (1+x) \geqslant x / 2$ and thus the inequality above implies

$$
j \leqslant \sqrt{2 \pi} \Delta^{2} n^{2.5} \cdot \ln \left(2^{n} / \operatorname{vol}\left(I_{0}\right)\right) .
$$


To finish the proof we need a lower bound on $\operatorname{vol}\left(I_{0}\right)$, i.e., the $n$-dimensional volume of the set $C_{v} \cap$ $B_{n}$. The normal cone $C_{v}$ contains the full-dimensional simplex spanned by 0 and the $n$ row-vectors $a_{i_{1}}, \ldots, a_{i_{n}}$ of $A$ that correspond to the inequalities of $A x \leqslant b$ that are tight at $v$. Since $A$ is integral, the volume of this simplex is at least $1 / n !$. Furthermore, if this simplex is scaled by $1 / \max \left\{\left\|a_{i_{k}}\right\|: k=\right.$ $1, \ldots, n\}$, then it is contained in the unit ball. Since each component of $A$ is itself a sub-determinant, one has $\max \left\{\left\|a_{i_{k}}\right\|: k=1, \ldots, n\right\} \leqslant \sqrt{n} \Delta$ and thus $\operatorname{vol}\left(I_{0}\right) \geqslant 1 /\left(n ! \cdot n^{n / 2} \Delta^{n}\right)$. It follows that (1) implies $j=O\left(\Delta^{2} n^{3.5} \log n \Delta\right)$.

Remarks. The result of Dyer and Frieze [DF94] is also based on analyzing expansion properties via isoperimetric inequalities. It is our choice of normal cones as the natural geometric representation, and the fact that we only ask for volume expansion instead of expansion of the graph itself, that allows us to get a better bound. Expansion properties of the graphs of general classes of polytopes have also been studied elsewhere in the literature, e.g. [Kal91, Kai04].

\section{Organization of the paper}

The next section is devoted to a proof of the volume-expansion property, i.e., Lemma 1, The main tool that is used here is a classical isoperimetric inequality that states that among measurable subsets of a sphere with fixed volume, spherical caps have the smallest circumference. Section 3 deals with unbounded polyhedra. Compared to the case of polytopes, the problem that arises here is the fact that the union of the normal cones is not the complete space $\mathbb{R}^{n}$. To tackle this case, we rely on an isoperimetric inequality of Lovász and Simonovits [LS93]. Finally, we discuss how our bound can be further generalized. In fact, not all sub-determinants of $A$ need to be at most $\Delta$ but merely the entries of $A$ and the $(n-1)$-dimensional sub-determinants have to be bounded by $\Delta$, which yields a slightly stronger result.

\section{Volume expansion}

This section is devoted to a proof of Lemma 1 Throughout this section, we assume that $P=\{x \in$ $\mathbb{R}^{n}: A x \leqslant b$ \} is a polytope. We begin with some useful notation. A (not necessarily convex) cone is a subset of $\mathbb{R}^{n}$ that is closed under the multiplication with non-negative scalars. The intersection of a cone with the unit ball $B_{n}$ is called a spherical cone. Recall that $C_{v}$ denotes the normal cone of the vertex $v$ of $P$. We denote the spherical cone $C_{v} \cap B_{n}$ by $S_{v}$ and, for a subset $U \subseteq V$, the spherical cone $\cup_{v \in U} S_{v}$ by $S_{U}$. Our goal is to show that the following inequality holds for each $I \subseteq V$ with $\operatorname{vol}\left(S_{I}\right) \leqslant \frac{1}{2} \operatorname{vol}\left(B_{n}\right)$ :

$$
\operatorname{vol}\left(S_{\mathscr{N}(I)}\right) \geqslant \sqrt{\frac{2}{\pi}} \frac{1}{\Delta^{2} n^{2.5}} \cdot \operatorname{vol}\left(S_{I}\right) .
$$

Recall that two vertices are adjacent in $G_{P}$ if and only if their normal cones have a common facet. This means that the neighbors of $I$ are those vertices $u$ for which $S_{u}$ has a facet which is part of the surface of the spherical cone $S_{I}$. In an iteration of breadth-first-search we thus augment the set of discovered vertices $I$ by those vertices $u$ that can "dock" on $S_{I}$ via a common facet. We call the $(n-1)$ dimensional volume of the surface of a spherical cone $S$ that is not on the sphere, the dockable surface $D(S)$, see Figure1,

The base of $S$ is the intersection of $S$ with the unit sphere. We denote the area of the base by $B(S)$. By area we mean the $(n-1)$-dimensional measure of some surface. Furthermore, $L(S)$ denotes the length of the relative boundary of the base of $S$. We use the term length to denote the measure of an $(n-2)$-dimensional volume, see Figure1, 


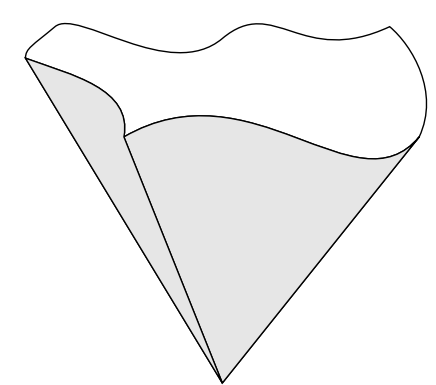

(a) Dockable surface of $S$.

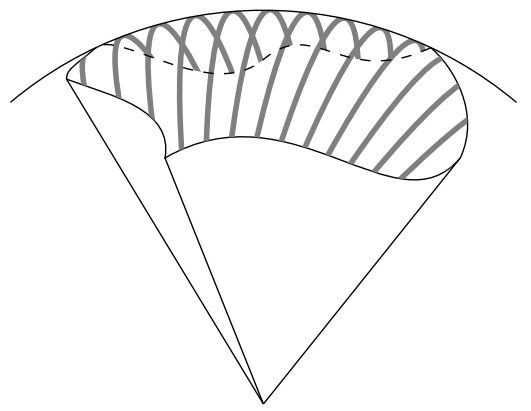

(b) Base of $S$.

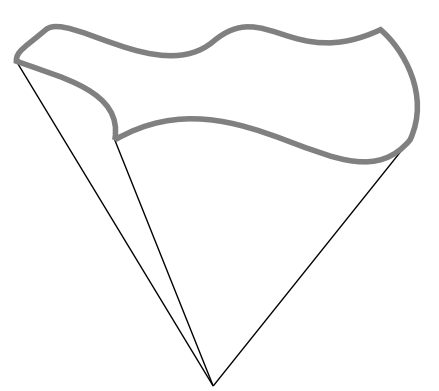

(c) Relative boundary of the base of $S$.

Figure 1: Illustration of $D(S), B(S)$ and $L(S)$.

Given any spherical cone $S$ in the unit ball, the following well-known relations follow from basic integration:

$$
\operatorname{vol}(S)=\frac{B(S)}{n}, \quad D(S)=\frac{L(S)}{n-1} .
$$

To obtain the volume expansion relation (2) we need to bound the dockable surface of a spherical cone from below by its volume and, for a simplicial spherical cone, we need an upper bound on the dockable surface by its volume. More precisely, we show that for every simplicial spherical cone $S_{v}$ one has

$$
\frac{D\left(S_{v}\right)}{\operatorname{vol}\left(S_{v}\right)} \leqslant \Delta^{2} n^{3}
$$

and for any spherical cone one has

$$
\frac{D(S)}{\operatorname{vol}(S)} \geqslant \sqrt{\frac{2 n}{\pi}} .
$$

Once inequalities (4) and (5) are derived, the bound (2) can be obtained as follows. All of the dockable surface of $S_{I}$ must be "consumed" by the neighbors of $I$. Using (5) one has thus

$$
\sum_{\nu \in \mathscr{N}(I)} D\left(S_{v}\right) \geqslant D\left(S_{I}\right) \geqslant \sqrt{\frac{2 n}{\pi}} \cdot \operatorname{vol}\left(S_{I}\right) .
$$

On the other hand, (4) implies

$$
\sum_{\nu \in \mathscr{N}(I)} D\left(S_{v}\right) \leqslant \Delta^{2} n^{3} \cdot \sum_{\nu \in \mathscr{N}(I)} \operatorname{vol}\left(S_{\nu}\right)=\Delta^{2} n^{3} \cdot \operatorname{vol}\left(S_{\mathscr{N}(I)}\right)
$$

These last two inequalities imply inequality (2). The remainder of this section is devoted to proving (4) and (5).

\subsection{Area to volume ratio of a spherical simplicial cone}

We will first derive inequality (4).

Lemma 3. Let $v$ be a vertex of P. One has

$$
\frac{D\left(S_{v}\right)}{\operatorname{vol}\left(S_{v}\right)} \leqslant \Delta^{2} n^{3} .
$$




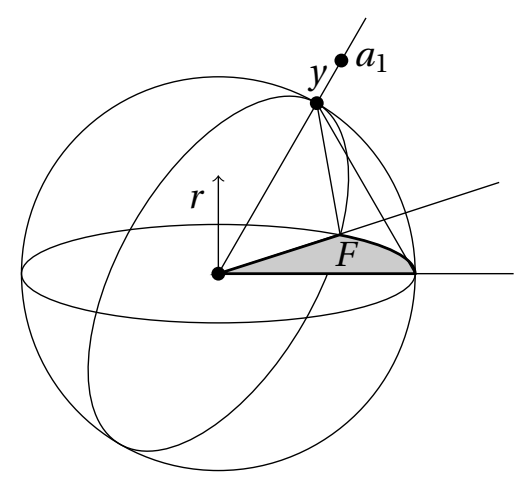

Figure 2: Proof of Lemmaß,

Proof. Let $F$ be a facet of a spherical cone $S_{v}$. Let $y$ be the vertex of $S_{v}$ not contained in $F$. Let $Q$ denote the convex hull of $F$ and $y$ (see Figure2). We have $Q \subseteq S_{\nu}$ because $S_{v}$ is convex. Moreover, if $h_{F}$ is the Euclidean distance of $y$ from the hyperplane containing $F$, then

$$
\operatorname{vol}\left(S_{v}\right) \geqslant \operatorname{vol}(Q)=\frac{\operatorname{area}(F) \cdot h_{F}}{n} .
$$

Summing over the facets of $S_{v}$, we find

$$
\frac{D\left(S_{v}\right)}{\operatorname{vol}\left(S_{v}\right)}=\sum_{\text {facet } F} \frac{\operatorname{area}(F)}{\operatorname{vol}\left(S_{v}\right)} \leqslant n \cdot \sum_{\text {facet } F} \frac{1}{h_{F}} .
$$

It remains to provide a lower bound on $h_{F}$. Let $a_{1}, \ldots, a_{n}$ be the row-vectors of $A$ defining the extreme rays of the normal cone of $v$, and let $A_{\nu}$ be the non-singular matrix whose rows are $a_{1}, \ldots, a_{n}$. Furthermore, suppose that the vertex $y$ lies on the ray generated by $a_{1}$. Let $H$ be the hyperplane generated by $a_{2}, \ldots, a_{n}$. The distance $d(y, H)$ of $y$ to $H$ is equal to $d\left(a_{1}, H\right) /\left\|a_{1}\right\|$. Let $b_{1}, \ldots, b_{n}$ be the columns of the adjugate of $A_{v}$. The column-vector $b_{1}$ is integral and each component of $b_{1}$ is bounded by $\Delta$. Furthermore $b_{1}$ is orthogonal to each of $a_{2}, \ldots, a_{n}$. Thus $d\left(a_{1}, H\right)$ is the length of the projection of $a_{1}$ to $b_{1}$, which is $\left|\left\langle a_{1}, b_{1}\right\rangle\right| /\left\|b_{1}\right\| \geqslant 1 /(\sqrt{n} \cdot \Delta)$, since $a_{1}$ and $b_{1}$ are integral. Thus

$$
h_{F}=d(y, H) \geqslant \frac{1}{n \Delta^{2}} .
$$

Plugging this into (8) completes the proof.

\subsection{An isoperimetric inequality for spherical cones}

We now derive the lower bound (5) on the area to volume ratio for a general spherical cone. To do that, we assume that the spherical cone has the least favorable shape for the area to volume ratio and derive the inequality for cones of this shape. Here one uses classical isoperimetric inequalities. The basic isoperimetric inequality states that the measurable subset of $\mathbb{R}^{n}$ with a prescribed volume and minimal area is the ball of this volume. In this paper, we need Lévy's isoperimetric inequality, see e.g. [FLM77, Theorem 2.1], which can be seen as an analogous result for spheres: it states that a measurable subset of the sphere of prescribed area and minimal boundary is a spherical cap.

A spherical cone $S$ is a cone of revolution if there exist a vector $v$ and an angle $0<\theta \leqslant \pi / 2$ such that $S$ is the set of vectors in the unit ball that form an angle of at most $\theta$ with $v$ :

$$
S=\left\{x \in B_{n}: \frac{v^{T} x}{\|v\|\|x\|} \geqslant \cos \theta\right\} .
$$




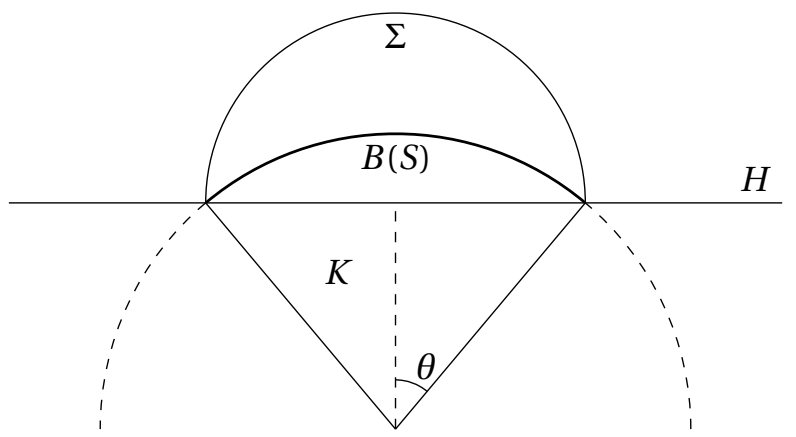

Figure 3: Proof of Lemma 5 .

Note that a spherical cone is a cone of revolution if and only if its base is a spherical cap. We also observe that two spherical cones of revolution, defined by two different vectors but by the same angle, are always congruent. Therefore, in the following we will only specify the angle of a cone of revolution.

Lemma 4. The spherical cone of given volume with minimum lateral surface is a cone of revolution.

Proof. By the first equation of (3), every spherical cone of volume $V$ intersects the unit sphere in a surface of area $n V$. Furthermore, by the second equation of (3), the length of the boundary of this surface is proportional to the area of the lateral surface of the cone. Then the problem of finding the spherical cone of volume $V$ with the minimum lateral surface can be rephrased as follows: Find a surface of area $n V$ on the unit sphere having the boundary of minimum length. By Lévy's isoperimetric inequality for spheres, the optimal shape for such a surface is a spherical cap. As observed above, this corresponds to a cone of revolution.

Lemma 5. Let $S$ be a spherical cone of revolution of angle $0<\theta \leqslant \pi / 2$. Then

$$
\frac{D(S)}{\operatorname{vol}(S)} \geqslant \sqrt{\frac{2 n}{\pi}}
$$

Proof. Using (3), we have to show that

$$
\frac{L(S)}{B(S)} \geqslant \sqrt{\frac{2}{\pi}} \frac{n-1}{\sqrt{n}}
$$

This is done in two steps. We first prove that this ratio is minimal for $S$ being the half-ball, i.e., $\theta=\pi / 2$. Then we show that $\frac{L(S)}{B(S)} \geqslant \sqrt{\frac{2}{\pi}} \frac{n-1}{\sqrt{n}}$ holds for the half-ball.

Let $H$ be the hyperplane containing the boundary of the base of $S$. Then $H$ divides $S$ into two parts: a truncated cone $K$ and the convex hull of a spherical cap. The radius $r$ of the base of $K$ is bounded by one.

Consider now the half-ball that contains $B(S)$ and that has $H \cap B_{n}$ as its flat-surface, see Figure 3, and let $\Sigma$ denote the area of the corresponding half-sphere. One has $B(S) \leqslant \Sigma$ and thus

$$
\frac{L(S)}{B(S)} \geqslant \frac{L(S)}{\Sigma} \text {. }
$$

Now $\Sigma$ and $L(S)$ are the surface of an $(n-1)$-dimensional half-sphere of radius $r$ and the length of its boundary respectively. If we scale this half-sphere by a factor of $1 / r$, we obtain the unit half-ball and its length respectively. Since scaling by a factor of $1 / r$ increases areas by a factor of $1 / r^{n-1}$ and 
lengths by a factor of $1 / r^{n-2}$, we have that $\frac{L(S)}{\Sigma}$ is at least the length of the unit-half-ball divided by the area of the base of the half-ball.

Suppose now that $S$ is the half-unit-ball. We show that the inequality $L(S) / B(S) \geqslant \sqrt{\frac{2}{\pi}} \frac{n-1}{\sqrt{n}}$ holds. The base of $S$ is a half unit sphere and $L(S)$ is the length of the boundary of a unit ball of dimension $n-1$. Thus

$$
B(S)=\frac{n}{2} \frac{\pi^{n / 2}}{\Gamma\left(\frac{n}{2}+1\right)}, \quad L(S)=\frac{(n-1) \pi^{(n-1) / 2}}{\Gamma\left(\frac{n-1}{2}+1\right)},
$$

where $\Gamma$ is the well-known Gamma function. Using the fact that $\Gamma(x+1 / 2) / \Gamma(x) \geqslant \sqrt{x-\frac{1}{4}}$ for all $x>\frac{1}{4}$ (see, e.g., |Mer96|), one easily verifies that

$$
\Gamma\left(\frac{n}{2}+1\right) \geqslant \sqrt{\frac{n}{2}} \cdot \Gamma\left(\frac{n-1}{2}+1\right) .
$$

It follows that

$$
\frac{L(S)}{B(S)}=\frac{2}{\sqrt{\pi}} \frac{n-1}{n} \frac{\Gamma\left(\frac{n}{2}+1\right)}{\Gamma\left(\frac{n-1}{2}+1\right)} \geqslant \sqrt{\frac{2}{\pi}} \cdot \frac{n-1}{\sqrt{n}} .
$$

Finally we are now ready to consider the case of an arbitrary spherical cone.

Lemma 6. Let $S$ be a (not necessarily convex) spherical cone with $\operatorname{vol}(S) \leqslant \frac{1}{2} \operatorname{vol}\left(B_{n}\right)$. Then

$$
\frac{D(S)}{\operatorname{vol}(S)} \geqslant \sqrt{\frac{2 n}{\pi}} .
$$

Proof. Let $S^{*}$ be a spherical cone of revolution with the same volume as $S$. By Lemma勾, $D(S) \geqslant D\left(S^{*}\right)$. Now, using Lemma 5 one has

$$
\frac{D(S)}{\operatorname{vol}(S)} \geqslant \frac{D\left(S^{*}\right)}{\operatorname{vol}\left(S^{*}\right)} \geqslant \sqrt{\frac{2 n}{\pi}} .
$$

This was the final step in the proof of Lemma[1]and thus we have also proved Theorem 2 , our main result on polytopes. The next section is devoted to unbounded polyhedra.

\section{The case of an unbounded polyhedron}

If the polyhedron $P$ is unbounded, then the union of the normal cones of all vertices of $P$ forms a proper subset $K^{\prime}$ of $\mathbb{R}^{n}$ : namely, $K^{\prime}$ is the set of objective functions $c$ for which the linear program $\max \left\{c^{T} x: x \in P\right\}$ has finite optimum. Similarly, the set $K^{\prime} \cap B_{n}$ is a proper subset of $B_{n}$. Then, given the union of the spherical cones that have already been discovered by the breadth-first-search (we denote this set by $S$ ), we should redefine the dockable surface of $S$ as that part of the lateral surface of $S$ that is shared by some neighboring cones. In other words, we should exclude the part lying on the boundary of $K^{\prime} \cap B_{n}$. However, this implies that Lemma [ cannot be immediately applied.

To overcome this difficulty, we make use of the Lovász-Simonovits inequality, which we now recall. Below we use notation $d(X, Y)$ to indicate the Euclidean distance between two subsets $X, Y \subseteq \mathbb{R}^{n}$, i.e., $d(X, Y)=\inf \{\|x-y\|: x \in X, y \in Y\}$. Also, $[x, y]$ denotes the segment connecting two points $x, y \in \mathbb{R}^{n}$ (see Figure 4). 


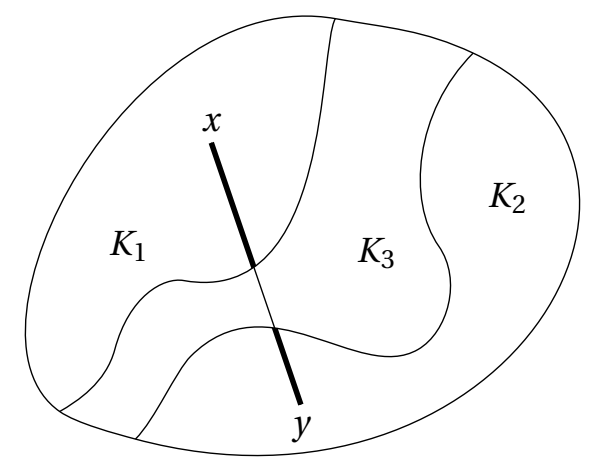

Figure 4: Illustration of the Lovász-Simonovits inequality.

Theorem 7. LS93 Let $K \subseteq \mathbb{R}^{n}$ be a convex compact set, $0<\varepsilon<1$ and $\left(K_{1}, K_{2}, K_{3}\right)$ be a partition of $K$ into three measurable sets such that

$$
\forall x, y \in K, \quad d\left([x, y] \cap K_{1},[x, y] \cap K_{2}\right) \geqslant \varepsilon \cdot\|x-y\| .
$$

Then

$$
\operatorname{vol}\left(K_{3}\right) \geqslant \frac{2 \varepsilon}{1-\varepsilon} \min \left(\operatorname{vol}\left(K_{1}\right), \operatorname{vol}\left(K_{2}\right)\right) .
$$

We now illustrate how the above result can be used in our context. Let $K=K^{\prime} \cap B_{n}$ and observe that $K$ is a convex and compact set. Let $S \subseteq K$ be the union of the spherical cones that have already been discovered by the breadth-first-search. We define the dockable surface of $S$ as that part of the lateral surface of $S$ that is disjoint from the boundary of $K$. We denote by $D^{\prime}(S)$ the area of the dockable surface of $S$. We can prove the following analogue of Lemma[6;

Lemma 8. If $\operatorname{vol}(S) \leqslant \frac{1}{2} \operatorname{vol}(K)$, then $D^{\prime}(S) \geqslant \operatorname{vol}(S)$.

Proof. Let $F$ denote the dockable surface of $S$ (thus $D^{\prime}(S)$ is the area of $F$ ). For every $\varepsilon>0$ we define

$$
\begin{aligned}
& K_{3, \varepsilon}=\left(F+\varepsilon B_{n}\right) \cap K, \\
& K_{1, \varepsilon}=S \backslash K_{3, \varepsilon}, \\
& K_{2, \varepsilon}=K \backslash\left(K_{1, \varepsilon} \cup K_{3, \varepsilon}\right),
\end{aligned}
$$

where $X+Y$ denotes the Minkowski sum of two subsets $X, Y \in \mathbb{R}^{n}$, i.e., $X+Y=\{x+y: x \in X, y \in Y\}$. Clearly $\left(K_{1, \varepsilon}, K_{2, \varepsilon}, K_{3, \varepsilon}\right)$ is a partition of $K$ into three measurable sets. Furthermore, condition (10) is satisfied. Thus Theorem7 7 implies that

$$
\frac{\operatorname{vol}\left(K_{3, \varepsilon}\right)}{2 \varepsilon} \geqslant \frac{1}{1-\varepsilon} \min \left(\operatorname{vol}\left(K_{1, \varepsilon}\right), \operatorname{vol}\left(K_{2, \varepsilon}\right)\right) .
$$

We observe that

$$
\begin{aligned}
\operatorname{vol}\left(K_{2, \varepsilon}\right) & \geqslant \operatorname{vol}(K \backslash S)-\operatorname{vol}\left(K_{3, \varepsilon}\right) \\
& \geqslant \operatorname{vol}(S)-\operatorname{vol}\left(K_{3, \varepsilon}\right) \\
& \geqslant \operatorname{vol}\left(K_{1, \varepsilon}\right)-\operatorname{vol}\left(K_{3, \varepsilon}\right) .
\end{aligned}
$$

Combining those two inequalities, we find

$$
\frac{\operatorname{vol}\left(F+\varepsilon B_{n}\right)}{2 \varepsilon} \geqslant \frac{\operatorname{vol}\left(K_{3, \varepsilon}\right)}{2 \varepsilon} \geqslant \frac{1}{1-\varepsilon}\left(\operatorname{vol}\left(K_{1, \varepsilon}\right)-\operatorname{vol}\left(K_{3, \varepsilon}\right)\right) .
$$


By a well-known result in geometry (see, e.g., [Fed69],) as $\varepsilon$ tends to 0 the left-hand side of (11) tends to the area of $F$, which is precisely the dockable surface $D^{\prime}(S)$. Moreover, as $\varepsilon$ tends to $0, \operatorname{vol}\left(K_{3, \varepsilon}\right)$ tends to 0 and $\operatorname{vol}\left(K_{1, \varepsilon}\right)$ tends to $\operatorname{vol}(S)$. We conclude that $D^{\prime}(S) \geqslant \operatorname{vol}(S)$.

Following the same approach as that used for the case of a polytope, one can show the following result for polyhedra.

Theorem 9. Let $P=\left\{x \in \mathbb{R}^{n}: A x \leqslant b\right\}$ be a polyhedron, where all sub-determinants of $A \in \mathbb{Z}^{m \times n}$ are bounded by $\Delta$ in absolute value. Then the diameter of $P$ is bounded by $O\left(\Delta^{2} n^{4} \log n \Delta\right)$. In particular, if $A$ is totally unimodular, then the diameter of $P$ is bounded by $O\left(n^{4} \log n\right)$.

\section{Remarks}

\subsection{Which sub-determinants enter the bound?}

For simplicity, we have assumed that a bound $\Delta$ was given for the absolute value of all sub-determinants of $A$. However, our proof only uses the fact the the sub-determinants of size 1 (i.e., the entries of the matrix) and $n-1$ are bounded. Calling $\Delta_{1}$ (resp. $\Delta_{n-1}$ ) the bound on the absolute value of the entries of $A$ (resp. on the sub-determinants of $A$ of size $n-1$ ), one easily verifies that all the results discussed above remain essentially unchanged, except that the statement of Lemma 3 becomes

$$
\frac{D\left(S_{v}\right)}{\operatorname{vol}\left(S_{v}\right)} \leqslant \Delta_{1} \Delta_{n-1} n^{3}
$$

and the lower bound on $\operatorname{vol}\left(I_{0}\right)$ becomes

$$
\operatorname{vol}\left(I_{0}\right) \geqslant \frac{1}{n ! n^{n / 2} \Delta_{1}^{n}}
$$

This implies the following strengthened result:

Theorem 10. Let $P=\left\{x \in \mathbb{R}^{n}: A x \leqslant b\right\}$ be a polyhedron, where the entries of $A$ (respectively the subdeterminants of $A$ of size $n-1$ ) are bounded in absolute value by $\Delta_{1}$ (respectively $\Delta_{n-1}$ ). Then the diameter of $P$ is bounded by $O\left(\Delta_{1} \Delta_{n-1} n^{4} \log n \Delta_{1}\right)$. Moreover, if $P$ is a polytope, its diameter is bounded by $O\left(\Delta_{1} \Delta_{n-1} n^{3.5} \log n \Delta_{1}\right)$.

\subsection{A more general geometric setting}

Since our result was first announced in $\left[\mathrm{BDSE}^{+} 12\right]$, Brunsch and Röglin [BR13] have found an algorithm to compute a short path between two given vertices of a non-degenerate polyhedron $P=\{x \in$ $\left.\mathbb{R}^{n}: A x \leqslant b\right\}$ that runs in expected polynomial time in $n, m$ and $1 / \delta$, where $\delta$ is a lower bound on the sine of the angle of a row of $A$ to the subspace of $n-1$ other rows of $A$. The expected length of the path is $O\left(m n^{2} / \delta^{2}\right)$. If $A \in \mathbb{Z}^{m \times n}$, then $\delta \geqslant 1 /\left(\Delta_{1} \Delta_{n-1} n\right)$, where $\Delta_{1}$ and $\Delta_{n-1}$ are, as before, bounds on the absolute values of $1 \times 1$ and $(n-1) \times(n-1)$ sub-determinants.

Our proof technique applies in this setting as well. We have volume expansion since the normal cones cannot be too flat. The parameter $\delta$ is a measure for this flatness. In this setting, Lemma 1 reads as follows.

Lemma 11. Let $P=\left\{x \in \mathbb{R}^{n}: A x \leqslant b\right\}$ be a polytope and let $I \subseteq V$ be a set of vertices with $\operatorname{vol}(I) \leqslant$ $(1 / 2) \cdot \operatorname{vol}\left(B_{n}\right)$. Then the volume of the neighborhood of I is at least

$$
\operatorname{vol}(\mathscr{N}(I)) \geqslant \sqrt{\frac{2}{\pi}}\left(\delta / n^{1.5}\right) \cdot \operatorname{vol}(I)
$$


The proof is along the lines of the proof of Lemma_ 1 and by adapting Lemma 3 . Here one has now

$$
D\left(S_{v}\right) / \operatorname{vol}\left(S_{v}\right) \leqslant n^{2} / \delta .
$$

Theorem[2] in the geometric setting now becomes the following.

Theorem 12. Let $P=\left\{x \in \mathbb{R}^{n}: A x \leqslant b\right\}$ be a polytope where the sine of the angle of any row of $A$ to the subspace generated by $n-1$ other rows of $A$ is at least $\delta$. The diameter of $P$ is bounded by $O\left(n^{2.5} / \delta \cdot \ln (n / \delta)\right)$.

Again, the proof is along the lines of the proof of Theorem 2 where the volume of $S_{v}$ is now lower bounded by $\delta^{n-1} / n$ !. In fact, the diameter bound $O\left(n^{2.5} / \delta \cdot \ln (n / \delta)\right)$ holds already for non-degenerate polytopes where each $S_{v}$ contains a ball of radius $\delta$. For polyhedra, we obtain a bound of

$$
O\left(n^{3} / \delta \cdot \ln (n / \delta)\right)
$$

on the diameter.

\section{Acknowledgments}

This work was carried out while all authors were at EPFL (École Polytechnique Fédérale de Lausanne), Switzerland. The authors acknowledge support from the DFG Focus Program 1307 within the project "Algorithm Engineering for Real-time Scheduling and Routing" and from the Swiss National Science Foundation within the project "Set-partitioning integer programs and integrality gaps".

\section{References}

[Bal84] M. L. Balinski. The Hirsch conjecture for dual transportation polyhedra. Math. Oper. Res., 9(4):629633, 1984.

[Bar74] D. Barnette. An upper bound for the diameter of a polytope. Discrete Math., 10:9-13, 1974.

BDSE $\left.^{+} 12\right]$ N. Bonifas, M. Di Summa, F. Eisenbrand, N. Hähnle, and M. Niemeier. On sub-determinants and the diameter of polyhedra. In Proceedings of the 28th annual ACM symposium on Computational geometry, SoCG '12, pages 357-362, 2012.

[BR13] T. Brunsch and H. Röglin. Finding short paths on polytopes by the shadow vertex algorithm. In Automata, Languages, and Programming, pages 279-290. Springer, 2013.

[BvdHS06] G. Brightwell, J. van den Heuvel, and L. Stougie. A linear bound on the diameter of the transportation polytope. Combinatorica, 26(2):133-139, 2006.

[DF94] M. Dyer and A. Frieze. Random walks, totally unimodular matrices, and a randomised dual simplex algorithm. Mathematical Programming, 64(1, Ser. A):1-16, 1994.

[DLKOS09] J. A. De Loera, E. D. Kim, S. Onn, and F. Santos. Graphs of transportation polytopes. J. Combin. Theory Ser. A, 116(8):1306-1325, 2009.

[EHRR10] F. Eisenbrand, N. Hähnle, A. Razborov, and T. Rothvoß. Diameter of polyhedra: Limits of abstraction. Mathematics of Operations Research, 35(4):786-794, 2010.

[Fed69] H. Federer. Geometric Measure Theory. Springer, 1969.

[FLM77] T. Figiel, J. Lindenstrauss, and V. Milman. The dimension of almost spherical sections of convex bodies. Acta Mathematica, 139(1):53-94, 1977.

[Kai04] V. Kaibel. On the expansion of graphs of 0/1-polytopes. In The sharpest cut, MPS/SIAM Ser. Optim., pages 199-216. SIAM, Philadelphia, PA, 2004. 
[Kal91] G. Kalai. The diameter of graphs of convex polytopes and $f$-vector theory. In Applied geometry and discrete mathematics, volume 4 of DIMACS Ser. Discrete Math. Theoret. Comput. Sci., pages 387-411. Amer. Math. Soc., Providence, RI, 1991.

[KK92] G. Kalai and D. J. Kleitman. A quasi-polynomial bound for the diameter of graphs of polyhedra. Bull. Amer. Math. Soc. (N.S.), 26(2):315-316, 1992.

[KS10] E. D. Kim and F. Santos. An update on the Hirsch conjecture. Jahresber. Dtsch. Math.-Ver., 112(2):7398, 2010.

[KW67] V. Klee and D. W. Walkup. The $d$-step conjecture for polyhedra of dimension $d<6$. Acta Math. 133, pages 53-78, 1967.

[Lar70] D. G. Larman. Paths of polytopes. Proc. London Math. Soc. (3), 20:161-178, 1970.

[LS93] L. Lovász and M. Simonovits. Random walks in a convex body and an improved volume algorithm. Random structures \& algorithms, 4(4):359-412, 1993.

[Mer96] M. Merkle. Logarithmic convexity and inequalities for the gamma function. Journal of mathematical analysis and applications, 203(2):369-380, 1996.

[Nad89] D. Naddef. The Hirsch conjecture is true for (0,1)-polytopes. Mathematical Programming, 45:109110, 1989.

[Orl97] J. B. Orlin. A polynomial time primal network simplex algorithm for minimum cost flows. Mathematical Programming, 78(2, Ser. B):109-129, 1997. Network optimization: algorithms and applications (San Miniato, 1993).

[San12] F. Santos. A counterexample to the Hirsch conjecture. Ann. of Math. (2), 176(1):383-412, 2012.

[Ver09] R. Vershynin. Beyond Hirsch conjecture: walks on random polytopes and smoothed complexity of the simplex method. SIAM J. Comput., 39(2):646-678, 2009. 